\title{
Radiofrequency ablation in palliative supportive care: Early clinical experience
}

\author{
FRÉDÉRIC MARCHAL ${ }^{1}$, LAURENT BRUNAUD ${ }^{3}$, CHRISTOPHE BAZIN $^{4}$, HERVÉ BOCCACINI $^{4}$, \\ PHILIPPE HENROT $^{2}$, PHILIPPE TROUFLEAU ${ }^{2}$, IVAN KRAKOWSKI $^{1}$ and DENIS REGENT ${ }^{4}$ \\ ${ }^{1}$ Interdisciplinary Department of Supportive Care of Patients in Oncology, ${ }^{2}$ Department of Radiology, Centre Alexis Vautrin, \\ Regional Cancer Center, Avenue de Bourgogne; ${ }^{3}$ Department of General, Digestive and Endocrine Surgery C, CHU, \\ ${ }^{4}$ Department of Radiology, CHU, Brabois University Hospital, 54511 Vandoeuvre-Lès-Nancy, France
}

Received August 22, 2005; Accepted October 20, 2005

\begin{abstract}
We report our early experience with radiofrequency ablation (RFA) in palliative supportive care. The medical files of eight patients were retrospectively reviewed. Four patients had a renal tumor, and nephrectomy was contraindicated in each patient since they had a poor general status. The fifth patient had a local recurrence in the site of a previous nephrectomy with a pancreatic tail extension, and surgical resection was contraindicated because of abdominal carcinomatosis. Two other patients had bone metastasis, one with a painful metastasis of mammary carcinoma in the head of the humerus resistant to radiotherapy, and the other with metastasis of the tibia of cutaneous melanoma. The last patient had a local recurrence of a sacral chordoma. Management, outcomes and complications were evaluated with $13.1 \pm 0.3$ months follow-up. All five patients with renal carcinomas did not have local recurrence. The two patients treated for bone metastases had no pain 8 weeks after RFA and remained stable over time. One complication occurred 2 months after using the procedure to treat the chordoma, and this patient was hospitalized for a fistula between the sigmoid and hypogastric artery false aneurysm and subsequently died. In conclusion, RFA can be a safe and useful adjuvant treatment in supportive care or unresponsive cancer pain patients. However, the destruction of tumoral tissues in contact with sensitive structures using RFA should be done with caution due to potentially severe complications.
\end{abstract}

\section{Introduction}

Cancer-associated pain is often the most debilitating aspect of malignant disease and could be a difficult clinical problem

Correspondence to: Dr Frederic Marchal, Department of Surgery, Centre Alexis Vautrin, Av. de Bourgogne, 54511 Vandoeuvre-lèsNancy, France

E-mail: f.marchal@nancy.fnclcc.fr

Key words: radiofrequency ablation, palliative care, renal tumor, bone metastasis, chordoma to manage. Treating pain from metastatic disease is often palliative and can be limited in effectiveness (1). Radiofrequency ablation (RFA) has long been used to treat painful disorders such as trigeminal neuralgia or osteom osteoid (2), and recent developments in technology and image guidance have allowed this invasive technique to treat solid tumors $(1,3)$. The first application concerned unresectable liver tumors, but other studies have shown that this technique could offer a valuable treatment option for other unresectable tumors (3-5). We report our experience with the use of RFA in palliative supportive care.

\section{Materials and methods}

Patients. The study was undertaken with the approval of our Institutional Human Studies Committee, and written informed consent was obtained from all patients. Five patients were treated for renal carcinomas (Fig. 1). Two patients, a 75- and 79-year-old, had a primary tumor and were recused intervention owing to a poor general status. The third patient had a left nephrectomy for a tumor in 1987. In 1998, he had a contralateral tumor on the remaining kidney, and a partial nephrectomy was performed. Three years after this partial nephrectomy, a local recurrence on the remaining kidney was diagnosed. This patient, a 79-year-old, was contraindicated for intervention to avoid renal failure.

The fourth patient, a 52-year-old woman, had an alcoholic cirrhosis (child B) with oedemato-ascitic decompensation. She had a carcinomatosis from an ovarian tumor (stage III) and a tumor of $2 \mathrm{~cm}$ diameter in the right kidney. It was first decided to equilibrate cirrhosis by medical treatment (paracentesis and abstaining from alcohol), then treat the ovarian tumor with surgery and chemotherapy. The tumor of the kidney was destroyed 3 weeks before ovarian surgery by RFA. These four tumors ranged in diameter from 1.5 to $3.0 \mathrm{~cm}$ (average diameter, $2.5 \pm 0.4 \mathrm{~cm}$ ). The fifth patient, a 65 -yearold, had a local recurrence at the site of previous nephrectomy (diameter, $4 \mathrm{~cm}$ ) with an extension to the tail of the pancreas, causing invalidante pain. The surgery was contraindicated owing to an abdominal carcinomatosis.

Two patients had a bone metastasis. The first patient, a 67-year-old, had metastasis of mammary carcinoma in the head of the humerus (Fig. 2). She had two courses of 
a

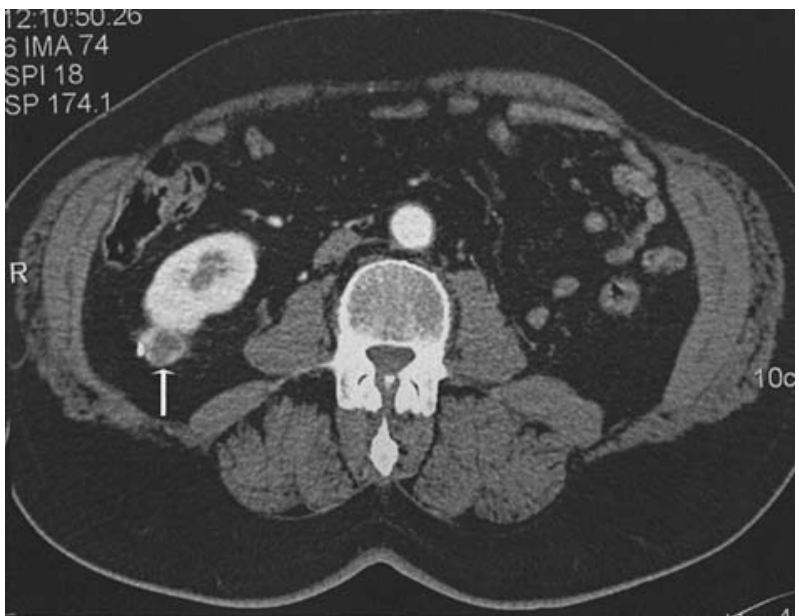

b

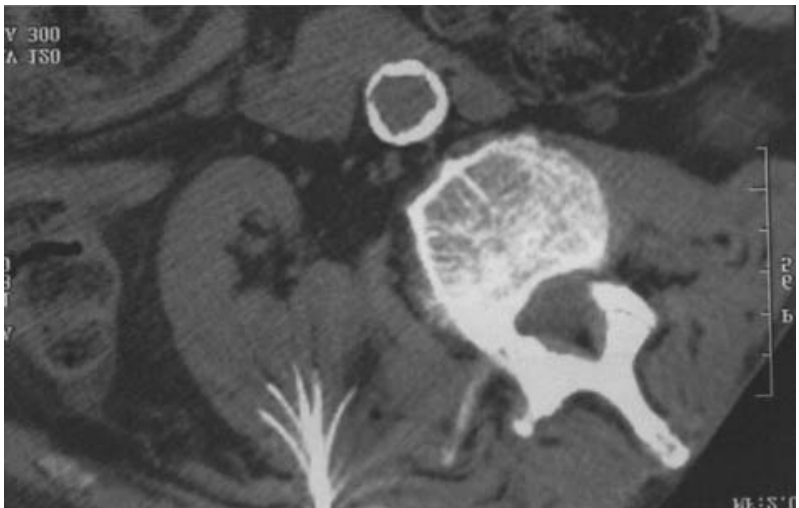

c

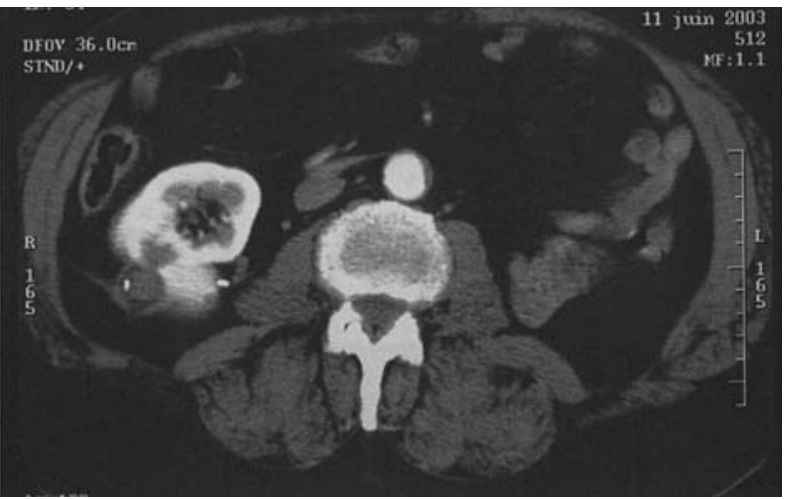

Figure 1. Small exophytic right renal tumor in a 79-year-old man, with a history of left nephrectomy for tumor and partial nephrectomy on the remaining kidney. (a) Preablation transverse CT scan with intravenous contrast material shows a $2.5 \mathrm{~cm}$ renal tumor (arrow) arising in the right kidney. (b) Unenhanced transverse CT scan shows the RFA probes in the tumor during treatment. (c) Transverse CT scan with intravenous contrast material 2 years after RFA shows a decrease in the size of the renal tumor. The residual tumor is not enhanced.

radiotherapy, but had painful impaired mobility one year after these treatments. The second patient had a 10-year history of cutaneous melanoma, with surgical ablation of 11 metatases and two medical treatments. She had metastasis of the tibia and 6 subcutaneous metastases. Melanoma being radio-resistant, it was decided to remove the subcutaneous metastases and treat the bone metastasis with RFA.

The last patient, a 49-year-old, had a sacral chordoma diagnosed in 1990, and three surgeries and two courses of radiotherapy (80 Gy) for local recurrences. He had a new local recurrence with pain (Fig. 3).

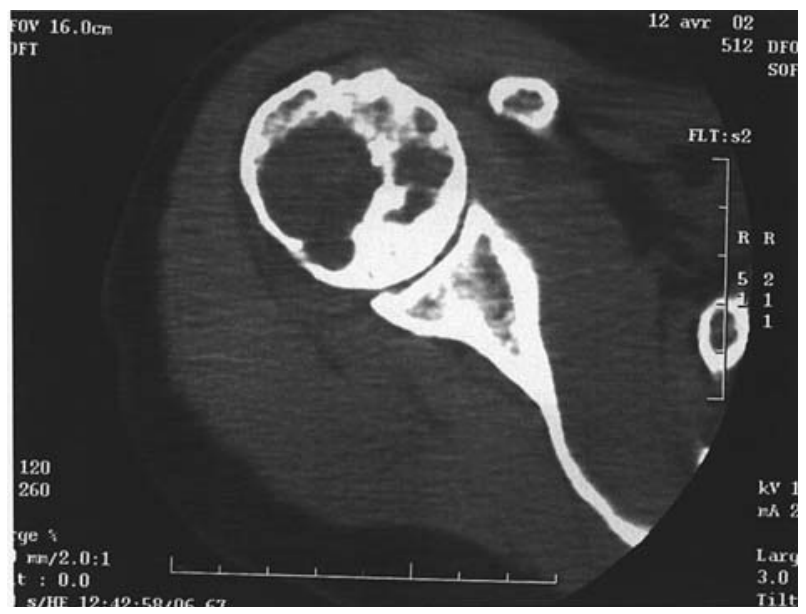

b

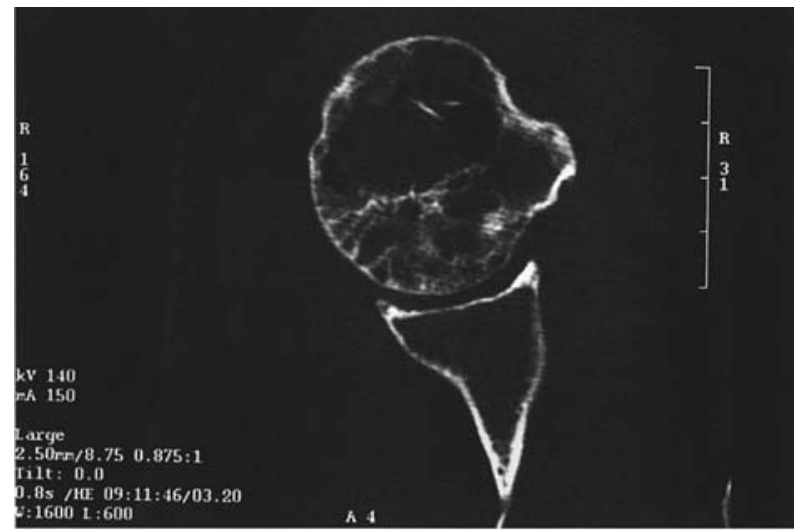

c

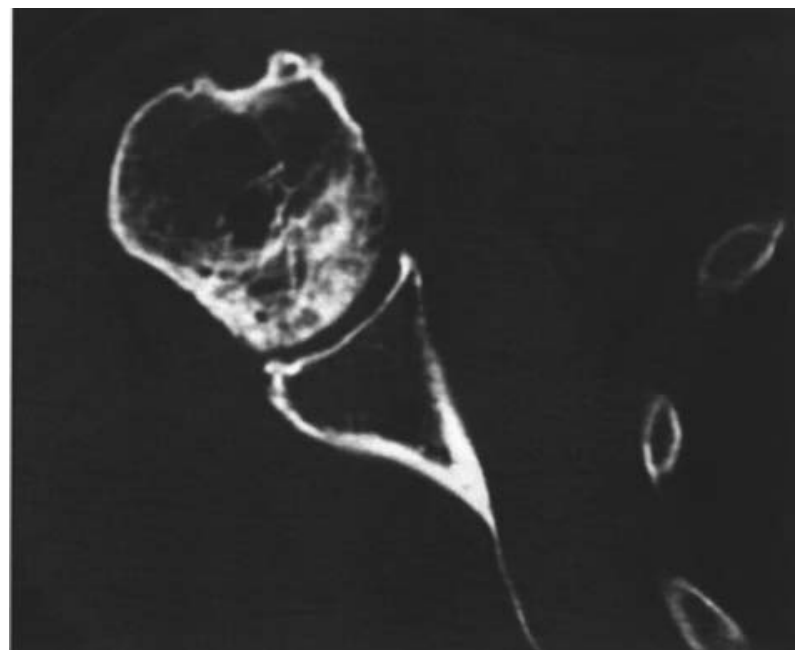

Figure 2. Proximal humeral metastasis from breast cancer in a 67-year-old woman. (a) Preablation transverse CT scan without intravenous contrast material shows a tumor in the head of the humerus. (b) Unenhanced transverse CT scan shows the RFA probes deployed in the tumor during treatment. (c) Transverse CT scan with intravenous contrast material 12 months after RFA shows modification of the tumor.

Radiofrequency ablation. For RFA, we used the RITA ${ }^{\circledR} 1500$ RF generator (RITA Medical Systems, Inc., Mountain View, CA, USA), which is a monopolar system rated at $460 \mathrm{kHz}$ $150 \mathrm{~W}$ at $50 \mathrm{ohms}$. Two dispersive electrodes were placed on the anterior face of the patient legs. All of the procedures were performed percutaneously under CT scan guidance (CT Pace ${ }^{\circledR}$; GE Medical Systems, Milwaukee, USA) (CT Twin ${ }^{\circledR}$; Philips, Eindhoven, The Netherlands), except for the chordoma in which a laparotomy was performed to dissect and protect 
a

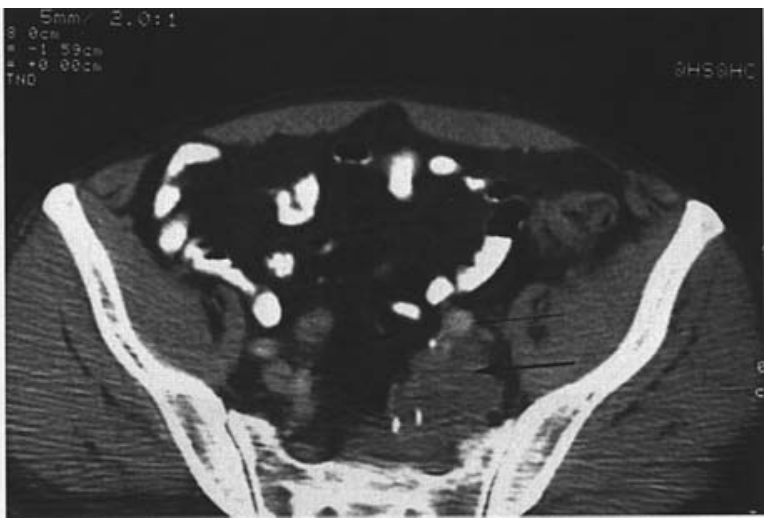

b

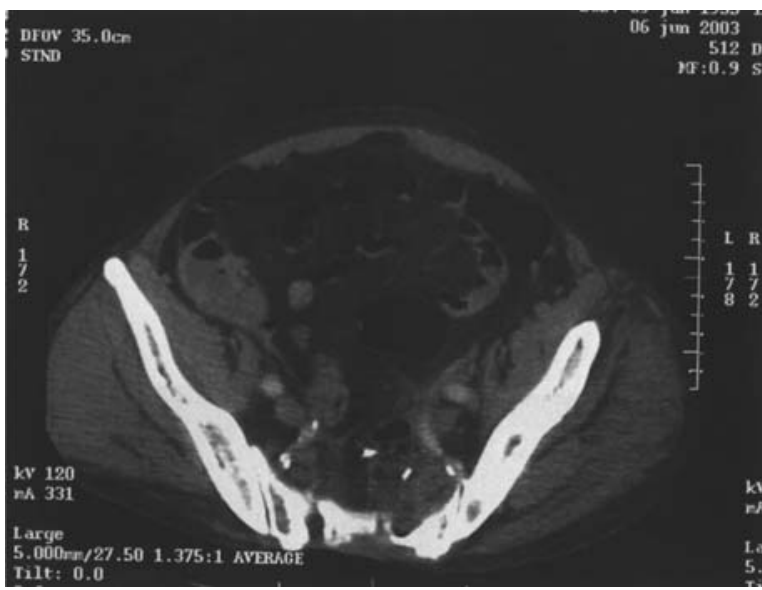

Figure 3. Sacral chordoma in a 49-year-old man. (a) Preablation transverse CT scan with intravenous contrast material shows a sacral chordoma (arrow) measuring $6 \times 4 \mathrm{~cm}$ in contact with the hypogastric artery (thin arrow). (b) Transverse CT scan with intravenous contrast material 1 month after RFA shows necrosis of the tumor.

the left ureter. Radiofrequency ablation of the chordoma was performed with real-time U.S. guidance (Logic $400 \mathrm{CL}^{\circledR}$; GE Medical Systems) by using a $7.5 \mathrm{mHz}$ scanning probe.

Assessment of treatment effectiveness. Initial post-treatment CT scans were obtained 1 month following ablation. Patients underwent subsequent follow-up with contrast materialenhanced CT scans at 3-4 month intervals during the first 2 years following ablation and thereafter at 6-month intervals. Areas of hypoattenuation not enhanced with contrast medium were considered to represent necrotic tissue.

Pain scoring. The degree of pain was evaluated with the visual analogue scale score (VAS score, 0-10) in patients who complained of pain. Patients were asked to rate their average pain during the past week $(0$, no pain; 10 , the worst imagined), then after the RFA procedure, during the first week and at 1 and 2 months.

Statistical analysis. Data are expressed as mean \pm SD. The VAS scores before treatment were compared with the scores obtained at 1 week, 1 and 2 months after treatment with non parametric Wilcoxon test. The data were analyzed and compared using Staview 5.0 ${ }^{\circledR}$ (Abacus Concepts, Berkeley, CA, USA). A p-value $<0.05$ was considered to be statistically significant.

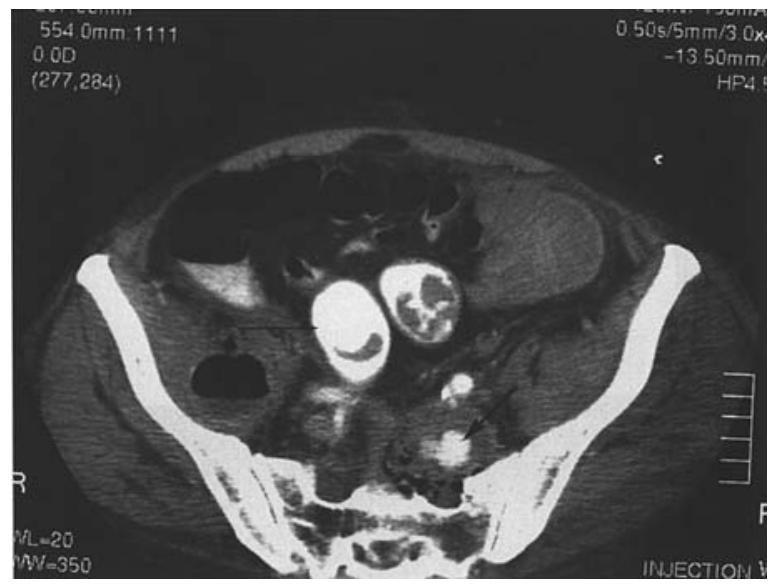

Figure 4. Sacral chordoma in a 49-year-old man. Patient was urgently hospitalized 2 months after RFA for rectorragies in a state of shock. Transverse CT scan with intravenous contrast material shows contrast in the tumor (arrow) and sigmoid colon (thin arrow) at the early arterial phase.

a

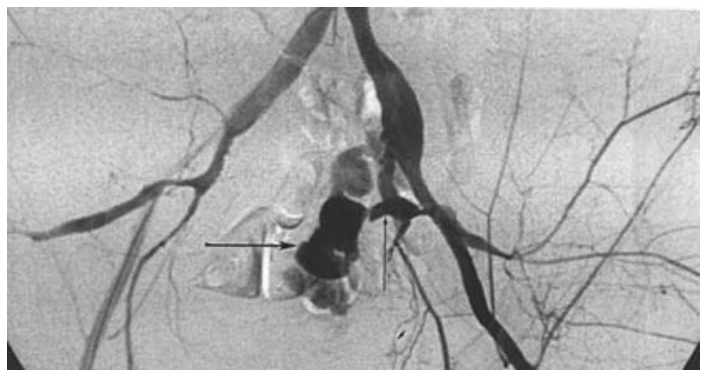

b

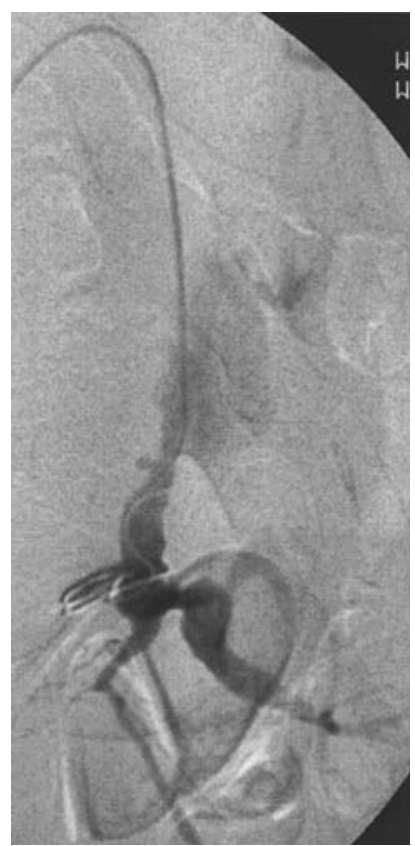

Figure 5. Sacral chordoma in a 49-year-old man. (a) Arteriography shows a fistula between the sigmoid (arrow) and hypogastric artery (thin arrow). (b) Arteriography after embolization of the arterial fistula by coils shows the occlusion of the fistula.

\section{Results}

The five patients with renal carcinomas did not have local recurrence. Four patients were alive at a follow-up of 6,15 , 19 and 23 months, and without renal failure. The fifth patient 
with carcinomatosis died 6 months after RFA. He had no pain after the procedure, and remained stable until his death. For the two patients treated for bone metastases, opiod requirements peaked at 1 week, then decreased over time. Eight weeks after RFA, patients had no pain and analgesic medication was unnecessary, and remained stable. The patient with metastasis of the humerus recovered mobility in his shoulder by his 24-month follow-up. CT scans did not show local recurrence with a follow-up of 12 (tibia metastasis) and 24 months (humerus metastasis), and pain created by the chordoma disappeared after RFA. A CT scan performed 1 month after the procedure showed a necrosis of the chordoma. Two months after the procedure, this patient was hospitalized for rectorragies with hemorrhagic shock. The arteriography showed at a fistula between the sigmoid and a false aneurysm of the hypogastric artery (Fig. 4). Despite an embolization that allowed the stabilization of the patient (Fig. 5), he died $5 \mathrm{~h}$ later from massive bleeding.

The patients with refractory pain ( 4 of 8 patients) reported reduced pain scores within 1 week, and 1 and 2 months after RFA, with a significant decrease in the mean VAS score from $7.5 \pm 1.7$ to $3.0 \pm 0.8$ at 1 week $(p=0.05)$, to $2.0 \pm 0.5$ $(\mathrm{p}=0.06)$ at 1 month and $1.5 \pm 0.6$ at 2 months $(\mathrm{p}=0.06)$.

\section{Discussion}

Radical nephrectomy is considered the standard treatment for renal carcinoma, and partial nephrectomy is an option for small localized renal carcinoma. Some patients, however, are not good candidates for surgical resection of their renal tumors because of comorbidities, limited functional renal reserve, or their history of disease (6). Since 1998, several clinical studies with relatively short-term follow-up have supported the efficacy of RFA to destroy renal carcinoma (7-10). The morbidity is low with approximately $1 \%$ serious complications (11), the most common being hematuria (12). Adjacent organs are protected from hyperthermia by fat tissue surrounding the kidney, and renal function is preserved by the procedure in our study. Tumor location and histologic characteristics, and the presence of renal insufficiency did not correlate with the success rate of RFA (6). The major determinant of successful complete ablation is tumor size, with a high success rate for tumors $<3 \mathrm{~cm}$ in diameter $(6,8)$. RFA was performed successfully for intractable hematuria after artery embolization had failed $(12,13)$. RFA was also used to treat unresectable local recurrence with success (14), as in our study. As resection of metastases of renal carcinoma infrequently results in improved prognosis, surgeons are often reluctant to perform this procedure. RFA could be an option, i.e. in pulmonary metastases (15). Because of low morbidity and extremely low risk of mortality associated with imaging-guided percutaneous RFA, this treatment may be considered for patients in whom systemic therapy has failed and the potential benefit appears to outweigh the risk of morbidity from metastasis resection (6).

Bone metastasis is a common problem in cancer patients and can cause pain and morbidity, particularly depression and anxiety (5). Treatment with external beam radiotherapy is the standard of care for patients with localized metastatic bone pain. However, the time required to achieve the maximum benefit after irradiation for metastatic lesions is usually 12-20 weeks, which is a lengthy time interval given the potentiallly brief life expectancy of these patients (16). Moreover, $20 \%$ to $30 \%$ of patients treated with radiotherapy do not experience pain relief and need opiod analgesics (16). Limitations include common recurrences, slow onset of relief, and tumor types that are less responsive to radiotherapy. We report a significant and rapid reduction in pain, similar to the study by Goetz et al, who found that $41 \%$ (17 of 41) of patients achieved at least a two-point drop in worst or average pain by week 1 , and $59 \%$ by week 4 (5). However, RFA is rarely used for bone metastases due to its radiosensitivity or patients presenting with diffuse bony metastatic disease and multiple painful sites (5). Lastly, lesions must be lytic or have a lytic component to expand the RFA tip into the metastasis (5). RFA of metastatic lesions involving bone is a safe procedure with complications less than 5\% (5). Using RFA to induce necrosis of the tumor could contribute to the development of a fracture, but a bone cement injection can be used in combination as prevention by stabilizing the ablated lesion within 1 week, especially in vertebral bones (17). The only contraindication to this technique is a destruction of the posterior vertebral body cortex, as the risk of causing thermal damage to the nerves is high (17).

Local recurrence of pelvic tumors may produce symptoms such as pain and often causes a marked deterioration in the quality of life and requires extreme caution in its treatment. Chemoradiotherapy affords limited clinical benefits, and aggressive surgery is often the only treatment; but patients whose conditions are indicated for surgical resection are limited. RFA could be an option for locally recurrent rectal cancer $(18,19)$ or chordoma, and has been used with success to treat recurrent sacral chordoma in 6 patients without complications (20-22). Chordomas are the most commonly occurring primary malignant spinal tumor, and more than half of primary sacral tumors are chordomas. The treatment for chordomas is debulking by surgery followed by adjuvant radiotherapy or proton therapy (20). However, chordomas have a high local recurrence rate, are slow-growing and cause low back pain and symptoms of mass that affect the quality of life of patients. RFA was performed for a recurrent chordoma and resulted in the relief of pain and tumor necrosis, but a fatal post-RFA complication occurred. It is considered that the vessels are protected from thermal damage by the vascular flow, which dissipates the heat generated in the ablated area ('cooling effect' or 'heat-sink effect') and protects the vessel walls (23). Previous surgical dissections and the high dose of pelvic radiotherapy (80 Gy) perhaps weakened the wall of the hypogastric artery and caused the complication. Tumor necrosis is the goal of treatment by RFA, but originated from complications in this case. Abcesses necessitating drainages and necrotizing pancreatitis with a hemorrhage are complications reported in literature $(19,24)$. Ohhigashi and Watanabe reported their experience with RFA for pelvic recurrence in 10 rectal carcinoma patients (18). Complications following RFA included 2 cases of abscess formation requiring percutaneous drainage, 2 cases of neuralgia and 1 case of arterial bleeding on day 27 post-RFA. Percutaneous embolization was 
performed through the internal iliac artery with success (18). The destruction of local tumors in contact with sensitive structures using RFA is not yet recommended because of potentially severe complications.

Cancer-related pain is a difficult problem, often with limited effective treatments, and pharmacologic management and opiates could cause sedation and may impair quality of life. Surgery is invasive, and repeat surgery can be difficult or morbid. Radiotherapy could be limited by the proximity of neural tissue, and maximum doses are often reached. RFA is a form of high temperature thermal therapy that induces coagulation necrosis, with imaging guidance ensuring accurate application of energy to the tumor, while minimizing collateral damage. The exact mechanism of action of thermal ablation for pain control is unknown (1). For direct nerve ablation, nociceptive input into the central nervous system is interrupted $(5,25,26)$; another potential mechanism is a decrease in intratumoral pressure, leading to decrease pressure on adjacent nerves $(1,27)$. By destroying tumor cells, RFA may decrease the production of cytokines and tumors factors involved in both nerve sensibilization and osteoclastic activity (5). RFA may also prevent the painful consequences of disease progression by inhibiting tumor growth (5). A multidisciplinary team approach should be employed when considering RFA for palliation, and consultation between the interventional radiologist, medical and/or surgical oncologist, and supportive care department members is ideal (27). The VAS scores before and after treatments were not statistically significant in our study due to the limits of a small patient population. RFA for palliation could be considered after conventional therapy failed, but with limited indications.

In conclusion, RFA can be a safe and useful adjuvant palliative treatment in supportive care or unresponsive cancer pain. RFA was accompanied by a significant reduction in pain and eliminated the need for pain medication. The use of RFA for the treatment of renal tumors should be considered for patients who are poor surgical candidates. RFA provides effective palliation of localized, painful osteolytic metastases involving bone. RFA may be an option for treating the local recurrence of pelvic tumors, but more studies are needed to validate its efficacy before it can be considered a palliative treatment.

\section{References}

1. Patti JW, Neeman Z and Wood BJ: Radiofrequency ablation for cancer-assiociated pain. J Pain 3: 471-473, 2002.

2. Albisinni U, Rimondi E, Malaguti MC and Ciminari R: Radiofrequency thermoablation in the treatment of osteoid osteoma. Radiology 232: 304-305, 2004.

3. Mirza AN, Fornage BD, Sneige N, Kuerer HM, Newman LA, Ames FC and Singletary SE: Radiofrequency ablation of solid tumors. Cancer J 7: 95-102, 2001.

4. Lencioni R, Crocetti L, Cioni R, Mussi A, Fontanini G, Ambrogi M, Franchini C, Cioni D, Fanucchi O, Gemignani R, Baldassarri R, Angeletti CA and Bartolozzi C: Radiofrequency ablation of lung malignancies: where do we stand? Cardiovasc Intervent Radiol 27: 581-590, 2004

5. Goetz MP, Callstrom MR, Charbonneau JW, Farell MA, Maus TP, Welch TJ, Wong GY, Sloan JA, Petersen IA, Beres RA, Regge D, Capanna R, Saker MB, Grönemeyer DHW, Gevardez A, Ahrar K, Choti MA, de Baere TJ and Rubin J: Percutaneous image-guided radiofrequency ablation of painful metastases involving bone: a multicenter study. J Clin Oncol 22: 300-306, 2004.
6. Zagoria RJ: Imaging-guided radiofrequency ablation of renal masses. Radiographics 24: S59-71, 2004.

7. Gervais DA, McGovern FJ, Wood BJ, Goldberg SN, McDougal WS and Mueller PR: Radiofrequency ablation of renal cell carcinoma: early clinical experience. Radiology 217 : 665-672, 2000.

8. Gervais DA, McGovern FJ, Arellano RA and Mueller PR: Renal cell carcinoma: clinical experience and technical success with radio-frequency ablation of 42 tumors. Radiology 226: 417-424, 2003.

9. Ogan K, Jacomides L, Dolmatch BL, Rivera FJ, Dellaria MF, Josephs SC and Cadeddu JA: Percutaneous radiofrequency ablation of renal tumors: technique, limitations, and morbidity. Urology 60: 954-958, 2002.

10. Su LM, Jarrett TW, Chan DY, Kavoussi LR and Solomon SB: Percutaneous computed tomography-guided radiofrequency ablation of renal masses in high surgical risk patients: preliminary results. Urology 61: 26-33, 2003.

11. Hines-Peralta A and Goldberg SN: Review of radiofrequency ablation for renal cell carcinoma. Clin Cancer Res 10: 6328S-6334S, 2004.

12. Rhim H, Dodd GD III, Chintapalli KN, Wood BJ, Dupuy DE, Hvizda JL, Sewell PE and Goldberg SN: Radiofrequency thermal ablation of abdominal tumors: lessons learned from complications. Radiographics 24: 41-52, 2004.

13. Wood BJ, Grippo J and Pavlovich CP: Percutaneous radio frequency ablation for hematuria. J Urol 166: 2303-2304, 2001.

14. McLaughlin CA, Chen MY, Torti FM, Hall MC and Zagoria RJ: Radiofrequency ablation of isolated local recurrence of renal cell carcinoma after radical nephrectomy. AJR Am J Roentgenol 181: 93-94, 2003.

15. Friedel G, Hurtgen M, Penzenstadler M, Kyriss T and Toomes H: Resection of pulmonary metastases from renal cell carcinoma. Anticancer Res 19: 1593-1596, 1999.

16. Janjan NA: Radiation for bone metastases: conventional techniques and the role of systemic radiopharmaceuticals. Cancer 80: 1628-1645, 1997.

17. Nakatsuka A, Yamakado K, Maeda M, Yasuda M, Akeboshi M, Takaki H, Hamada A and Takeda K: Radiofrequency ablation combined with bone cement injection for the treatment of bone malignancies. J Vasc Interv Radiol 15: 707-712, 2004.

18. Ohhigashi S and Watanabe F: Radiofrequency ablation is useful for selected cases of pelvic recurrence of rectal carcinoma. Tech Coloproctol 7: 186-191, 2003.

19. Kalil AN, Santarosa F, Cunha L, Beck P, Riccardi F, Cruz JV and Vinholes J: Radiofrequency ablation in the treatment of pelvic recurrence of rectal cancer. Hepatogastroenterology 50: 1937-1939, 2003.

20. Neeman Z, Patti JW and Wood BJ: Percutaneous radiofrequency ablation of chordoma. AJR Am J Roentgenol 179: 1330-1332, 2002.

21. Albisinni U, Rimondi E, Malaguti MC and Ciminari R: Percutaneous radiofrequency ablation of chordoma. AJR Am J Roentgenol 183: 245-246, 2004.

22. Anis N, Chawki $\mathrm{N}$ and Antoine K: Use of radiofrequency ablation for the palliative treatment of sacral chordoma. AJNR Am J Neuroradiol 25: 1589-1591, 2004.

23. De Baere T, Denys A, Wood BJ, Kardache M, Vilgrain V, Menu Y and Roche A: Radiofrequency liver ablation: experimental comparative study of water-cooled versus expandable systems. AJR 176: 187-192, 2001.

24. Elias D, Baton O, Sideris L, Lasser P and Pocard M: Necrotizing pancreatitis after radiofrequency destruction of pancreatic tumours. Eur J Surg Oncol 30: 85-87, 2004.

25. Kapural L and Mekhail N: Radiofrequency ablation for chronic pain control. Curr Pain Headache Rep 5: 517-525, 2001.

26. Evrard S: Surgical lesioning of splanchnic nerves using wet needle radiofrequency thermoablation. J Surg Oncol 80: 171-172, 2002.

27. Locklin JK, Mannes A, Berger A and Wood BJ: Palliation of soft tissue cancer pain with radiofrequency ablation. J Support Oncol 2: 439-445, 2004. 